Teil I: Erstes Hauptstück: Leben, Lesen und Lieben im Abendland 



\section{Einführung in die Themenstellung der Vorlesung}

Unsere Vorlesung versucht, einem verführerisch vielfältigen und unendlich verwirrenden Thema näher und auf die Schliche zu kommen: der Liebe. Zugleich einer Tätigkeit und kulturellen Praxis, die Literatur erst zum Leben erweckt: dem Lesen. Dies ist eine waghalsige, riskante, aber eben darum auch attraktive und überaus verlockende Themenstellung, der wir uns in der Folge liebevoll und mit der notwendigen literaturwissenschaftlichen Schärfe und vor allem Würze widmen werden, ganz dem Schlusswort von Roland Barthes in seiner Leçon vor dem Collège de France folgend: „Sapientia: nul pouvoir, un peu de savoir, un peu de sagesse, et le plus de saveur possible." ${ }^{1}$ Und an Würze, an saveur, wird es in dieser Vorlesung nicht mangeln!

Dabei geht es im Kern um die spezifisch literaturwissenschaftlichen und ästhetischen Implikationen eines Lebenswissens, das als diskursiver Treibstoff und erzählerische Bewegungsfigur die Literaturen (nicht erst) der Moderne in Gang hält. Von der Liebe und dem Abendland (Denis de Rougemont) bis zu den Fragmenten einer Sprache der Liebe (Roland Barthes), den Schreibformen des Marquis de Sade, von Giacomo Casanova oder Italo Calvino über die Liebes- und Lesekristallisationen Prousts bis hin zur Liebe in den Zeiten der Cholera (Gabriel García Márquez), von der Liebe zwischen zwei Dichtern und der Transzendenz ihres Begehrens (Juana Borrero) zu den Liebesgeschichten zwischen den Sprachen und Kulturen (Assia Djebar), hin zu den Theorien der Liebesgeschichten nach der Liebe (Michel Houellebecq), von Tristan und Isolde über Don Juan sowie Romeo und Julia bis zu Sab, Emma Bovary und den Versatzstücken aktueller Massenkommunikation zwischen Lese- und Liebesrevolution soll das Verhältnis von Liebe, Leben und Lesen, von Literatur und Leidenschaft analysiert werden. Ziel unserer Vorlesung ist nicht die Definition von Liebe, sondern ein Parcours durch grundlegende Konstellationen literarischer Leidenschaft unter den Bedingungen der Moderne mit Vorliebe, aber keineswegs ausschließlich in den Romanischen Literaturen der Welt.

Eine Vorlesung über die Liebe ist innerhalb der üblichen Lehrveranstaltungen im Rahmen der Romanistik oder über unser Fach hinaus an dieser Fakultät keine alltägliche und keine gewohnte, beziehungsweise gewöhnliche Angelegenheit. Die Themenstellung ,Liebe / Lesen“ ist etwas ganz Besonderes, auf Bayerisch würde man es wohl ein Schmankerl nennen, zumal sie gepaart ist mit einem speziellen Fokus auf dem Lesen und damit der aktiven Rolle der Lesenden

1 Barthes, Roland: Leçon. Leçon inaugurale de la Chaire de sémiologie littéraire du Collège de France, prononcée le 7 janvier 1977. Paris: Seuil 1978, S. 46.

Ә Open Access. (C) 2020 Ottmar Ette, publiziert von De Gruyter. (๕)BY-NC-ND Dieses Werk ist lizenziert unter der Creative Commons Attribution-NonCommercial-NoDerivatives 4.0 International Lizenz. 
(und implizit auch Liebenden). Lesen und Lieben verbinden sich - das werden wir noch sehen - in vielfältigen Figuren der Liebe miteinander: Und gerade darin ist auch ein nicht geringer Teil der diskursiven Triebkraft dieses Treibstoffes zu sehen.

Auch wenn wir in literaturwissenschaftlichen Seminaren selbstverständlich immer wieder auf das Thema Liebe stoßen, scheint dieser Themenbereich doch viel $\mathrm{zu}$ komplex, viel $\mathrm{zu}$ weitschweifig und allzu wenig fassbar, als dass wir der Liebe - und sei es nur jener in den Romanischen Literaturen der Welt in der Moderne - eine ganze Vorlesung widmen sollten oder könnten. Wird uns das Thema nicht etwa zwischen den Fingern zerrinnen? Werden wir überhaupt in der Lage sein, die Liebe begrifflich fassen zu können? Welche Kategorien und Einteilungen lassen sich entwickeln, um aus literatur- und kulturwissenschaftlicher Perspektive Herangehensweisen zu entwickeln, ein solch überwältigendes Thema zu bewältigen?

Die Liebesthematik ist zweifellos sehr anziehend und verführerisch, sind wir doch alle mit dieser literaturwissenschaftlichen Themenstellung oder viel besser: mit diesem großen Gefühl, das uns alle beschäftigt, bindet, verstrickt höchst verbandelt. Wenn wir über Liebe sprechen, sprechen wir vielleicht auch immer über unsere Liebe, mag sein. Darin liegt eine Gefahr, aber auch eine Chance, dies wird jeweils auszuloten sein. In jedem Falle aber reicht ein Leben nicht hin, alle Varianten und Variationen der Liebe auszukosten. Aber keine Bange: Dafür haben wir ja die Literaturen der Welt.

Im Verlauf unserer Vorlesung werden Sie sicherlich auch vieles darüber erfahren, was Sie noch nicht wussten. Aber auch sie weiß vieles von der Liebe nicht. Gerade darin auch will sie liebevoll vorgehen ... und wohldosiert. Denn nicht umsonst warnt uns William Shakespeare gleich zu Beginn von Romeo und Julia, dass alle Kräuter stets ein pharmakon seien: Genießen wir etwa nur ihren Duft, so wirken sie wohltuend und kräftigend; schlucken wir sie hinunter, so können sie uns umbringen. Nicht umsonst ist Liebe als eines der großen Gefühle gerade auch in diesem Sinne ein pharmakon: ${ }^{2}$ Es kommt stets auf den Gebrauch und nicht zuletzt auch auf die Dosierung an. Aber dringen wir nicht gleich zu Beginn unserer Vorlesung zu tief in die Texte und Praktiken ein.

Doch wie behandeln wir im Verhältnis zur Liebe das Lesen? Nun, noch einmal ein Anstoß aus dem anglophonen Raum. Virginia Woolf gab uns 1923 eine Leitlinie vor: „Denn das Verlangen zu lesen ist wie alle anderen Sehnsüchte, die

2 Vgl. hierzu Fuest, Leonhard: Materia Cruda. Zur Essentialität des Poetopharmakons. In: Ette, Ottmar / Sánchez, Yvette / Sellier, Veronika (Hg.): LebensMittel. Essen und Trinken in den Künsten und Kulturen. Zürich: Diaphanes 2013, S. 81-89; sowie (ders.): Poetopharmakon. Heilmittel und Gifte der Literatur. Bielefeld: Transcript Verlag 2015. 
unsere unglückliche Seele aufwühlen, der Analyse fähig. “3 Kaum ein Gegenstand der fremdsprachigen Philologien ist spannender und zugleich auch verwirrender als die Geschichte und die Geschichten des Lesens. Da tut es gut, aus berufenem Munde die Analysefähigkeit des Lesens attestiert zu bekommen. Und natürlich war und ist die Analyse des Lesens eine der vorzüglichsten Aufgaben von Literatur- und Kulturwissenschaft.

Literarische wie nicht-literarische Texte existieren erst als Werke, wenn es ein Lesepublikum - und sei es noch so klein - gibt, das sie distanziert liest, aufgeregt verschlingt und sie gerade dadurch erst in Bewegung setzt. Eine Literatur im abendländischen Sinne kann es ohne Leserinnen und Leser streng genommen nicht geben. Wir werden uns im Verlauf der Vor-Lesung mit verschiedensten Lese(r)figuren und Lesarten, mit der Geschichte des Lesens von der Schriftrolle bis zur Lektüre von Palindromen auf unserer DNS und vom Tod des Autors bis zur Geburt des Lesers, mit unterschiedlichsten Leseanweisungen in literarischen Texten, mit den Orten des Lesens zwischen Bibliothek, Bahn und Bett, mit dem lauten und dem stillen Lesen und seinen körper-leiblichen Konsequenzen, mit den Folgen des Lesens von der Verführung und Verdammung bis zur Verwissenschaftlichung oder Verdummung beschäftigen, wobei ungeachtet mancher Ausflüge der Schwerpunkt unserer Vorlesung wiederum auf den Romanischen Literaturen der Welt liegen wird. Die Liebe wird beim Lesen stets mit dabei sein.

Quer zur Geschichte und zur Theorie des Lesens als kultureller und sozialer, als politischer, ökonomischer und symbolischer Praxis sollen uns die Geschichten des Lesens und die Lust am Text in ihren Bann ziehen. Denn genau hier gibt es jene Überschneidung, die ich nicht zufällig zentral zu stellen versuchen werde: eine Art Kreuzungspunkt in der Lust am Text oder wie Roland Barthes es in einem eindrucksvollen Kurztext der Theorie formulierte: Le Plaisir du texte. ${ }^{4}$ Denn es ist diese Lust, welche die Liebe mit dem Lesen als Leidenschaft verbindet.

Doch es gibt noch eine zweite Verbindung, welche Liebe und Lesen neben der Lust am Text miteinander verbindet. Es ist ein viertes $L$, ein vierter Begriff, der auch in unserer Vorlesung eine gewichtige Rolle spielen wird: Es ist das Lebenswissen. Denn die Literatur ist aus meiner Sicht in einer ganz grundlegenden Weise ein sich wandelnder interaktiver Speicher von Lebenswissen, aus dem in den unterschiedlichsten Kulturen, Sprachen und Literaturen der Welt immer wieder ein Wissen über das Leben geschöpft wurde. Literatur also ist in einem ganz

3 „For the desire to read, like all the other desires which distract our unhappy souls, is capable of analysis." Woolf, Virginia: Sir Thomas Browne, a review of the Golden Cockerel edition of the Works of Sir Thomas Browne. In: Times Literary Supplement (June 28, 1923), S. 436.

4 Vgl. Barthes, Roland: Die Lust am Text. Aus dem Französischen von Ottmar Ette. Kommentar von Ottmar Ette. Berlin: Suhrkamp Verlag (Suhrkamp Studienbibliothek 19) 2010. 
grundlegenden Sinne Lebenswissen, aber auch Erlebenswissen, Überlebenswissen und Zusammenlebenswissen, ja selbst ein Weiterlebenswissen, obwohl wir diesen letzteren Punkt nur selten in unserer Vorlesung berühren werden.

Dies bedeutet freilich keineswegs, dass ich damit behaupten würde, man könnte die Literatur mit der außersprachlichen Realität oder schlicht dem tatsächlich gelebten Leben gleichsetzen. Nichts läge mir ferner. Aber mir scheint es weder historisch noch methodologisch gerechtfertigt, so zu tun, als hätte die Literatur mit dem Leben nichts zu schaffen. Dafür sprechen alle Befunde rund um die Literaturen der Welt eine ganz andere Sprache. Übrigens: Wenn ich von den Literaturen der Welt spreche, so meine ich dies in einem Sinne, der den Begriff der Weltliteratur Goethe'scher Provenienz als einen längst historisch gewordenen betrachtet. Aber davon später ein wenig mehr. ${ }^{5}$

Jener Bereich aber, in dem die Überschneidung von Literatur, Lieben und Leben sich ansiedelt, ist das Lesen, ist die Lektüre. Genau deshalb sollten wir uns aus einer veränderten Perspektivik an die Formen der Lektüre und des Lesens, aber auch - um mit dem Philosophen Hans Blumenberg zu sprechen - an die Frage einer Lesbarkeit der Welt und ihrer großen Emotionen neu annähern. Die Lektüre, das Lesen bildet daher den zweiten Mittelpunkt all jener Fragen, um die unsere aktuelle Vorlesung kreisen wird. Denn Hans Blumenberg hatte schon früh darauf hingewiesen, wie schon im altgriechischen Epos oder im Buch der Bücher die Bucherfahrung in Rivalität zur Welterfahrung tritt. Gleich zu Beginn seines ersten Kapitels, das unter dem Thema „Bücherwelt und Weltbuch“ steht, schrieb der deutsche Philosoph:

\begin{abstract}
Zwischen den Büchern und der Wirklichkeit ist eine alte Feindschaft gesetzt. Das Geschriebene schob sich an die Stelle der Wirklichkeit, in der Funktion, sie als das endgültig Rubrizierte und Gesicherte überflüssig zu machen. Die geschriebene und schließlich gedruckte Tradition ist immer wieder zur Schwächung von Authentizität der Erfahrung geworden. Es gibt so etwas wie die Arroganz der Bücher durch ihre bloße Quantität, die schon nach einer gewissen Zeit schreibender Kultur den überwältigenden Eindruck erzeugt, hier müsse alles stehen und es sei sinnlos, in der Spanne des ohnehin allzu kurzen Lebens noch einmal hinzusehen und wahrzunehmen, was einmal zur Kenntnis genommen und gebracht worden war. ${ }^{6}$
\end{abstract}

Sie sehen also, dass es einen lektüre- und buchtechnischen Hintergrund hatte, wenn ich Ihnen eingangs versicherte, dass wir alle in unserem je individuellen Leben niemals alle Liebespraktiken würden ausschöpfen können, die es gibt oder

5 Zum Begriff der Literaturen der Welt vgl. Ette, Ottmar: WeltFraktale. Wege durch die Literaturen der Welt. Stuttgart: J.B. Metzler Verlag 2017.

6 Blumenberg, Hans: Die Lesbarkeit der Welt. Frankfurt a. M.: Suhrkamp 1986, S. 17. 
die denkbar sind, aber dass wir zugleich dafür ja den großen Speicher der Literaturen der Welt hätten, welcher letztlich eine wahrlich erschöpfende Menge an Varianten und Variationen von Liebe enthalten würde - und tatsächlich auch enthält.

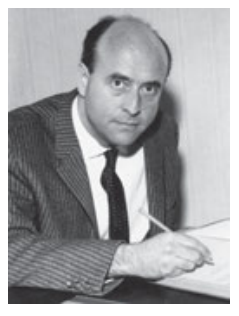

Abb. 1: Hans Blumenberg (Lübeck, 1920 - Altenberge bei Münster, 1996).

Lassen Sie mich hier auf eben diesen großen Speicher der Literaturen der Welt zurückgreifen und Ihnen eine der für mich selbst gewiss schönsten, ergreifendsten und zugleich hintergründigsten Passagen jener Literaturgeschichte präsentieren, in der wir selbst auf gewisse Weise immer noch leben. Denn in ihr treffen wir auf zwei Liebende, auf Paolo und Francesca, die in die Hölle kamen, weil sie gemeinsam ein Buch lasen - und sie taten dies, wie im Mittelalter eben üblich, mit lauter Stimme, bewegten also ihre Münder. Doch hören Sie selbst und lernen Sie dieses vielleicht berühmteste Liebespaar der Welt, das von der Erde verbannt wurde, kennen:

Per più fiate gli occhi ci sospinse quella lettura, e scolorocci il viso: ma solo un punto fu quel che ci vinse. Quando leggemmo il disiato riso esser baciato da cotanto amante, questi, che mai da me non fia diviso, la bocca mi baciò tutto tremante. Galeotto fu il libro e chi lo scrisse: quel giorno più non vi leggemmo avante.

[Mehrmals ließ, was wir da lasen, uns die Augen erheben; wir sahen uns ins bleiche Gesicht, aber dann kam eine einzige Stelle, die uns besiegte. Als wir lasen, wie der begehrte lachende Mund von diesem Liebhaber geküßt wurde, da küßte dieser Mann, der niemals von mir getrennt wird, mich auf den Mund, zitternd am ganzen Leib. Den Kuppler Galehaut spielten das Buch und der es schrieb. An diesem Tag lasen wir nicht weiter. $]^{7}$

7 Alighieri, Dante: Commedia, Inferno V, 130-138. Deutsche Übersetzung aus Alighieri, Dante: Commedia: In deutscher Prosa von Kurt Flasch. Frankfurt a. M.: S. Fischer 2011, Inferno, V, 130-138. 


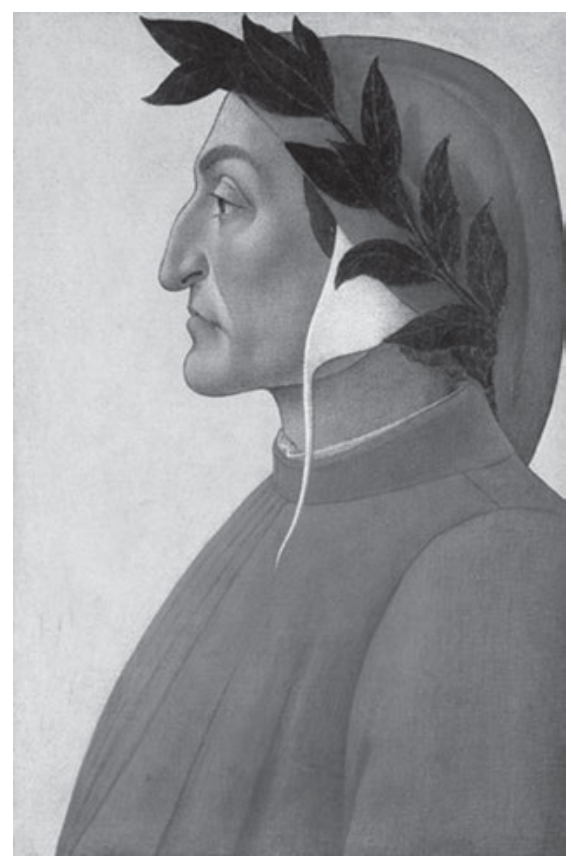

Abb. 2: Dante Alighieri (Florenz, 1265 Ravenna, 1321).

Es ist - und dies ist in den Literaturen der Welt fürwahr kein seltenes Thema - die Beschreibung einer Ehebruchszene, welche die beiden Liebenden mit nachfolgenden Höllenqualen teuer bezahlen müssen. Eine tragische Liebesbeziehung? Ja, zweifellos. Doch wie schön war die Liebe zwischen den beiden zuvor. Denn die gemeinsame Lektüre führt zu gemeinsamer Liebe, der Mund als Stimmbildungsort wird zum Mund, auf den sich ein Kuss senkt, bevor sich ein anderer Mund öffnet, der die beiden Liebenden miteinander vereint.

Der gefährliche Leseakt entbrannte an einem Text aus dem Lancelot-Stoff, an jener Figur von Galeotto, die wir mit dem edlen Galehaut aus dem Umkreis von Lancelot identifizieren und damit in der mittelalterlichen Erzähl- und Sagenwelt verorten können. Das gemeinsame Lesen verwandelt sich in ein gemeinsames Lieben, da die Worte des Dichters durch den Kuss ins Stocken kommen und durch die eigene Körpersprache, die Sprache der eigenen Körper, ersetzt werden. „An jenem Tage lasen sie nicht weiter“ - ist dies nicht die schönste aller Liebesbeschreibungen einer Liebe, die wohlgemerkt in die Hölle führt, aber zuvor den Umweg über das Liebesparadies einschlägt? Denn das Nicht-Weiterlesen eröffnet jenen Raum der Liebe, welcher die beiden Liebenden vereinen und zur Vereinigung führen wird. Wir werden Paolo und Francesca bald schon wiedersehen. 


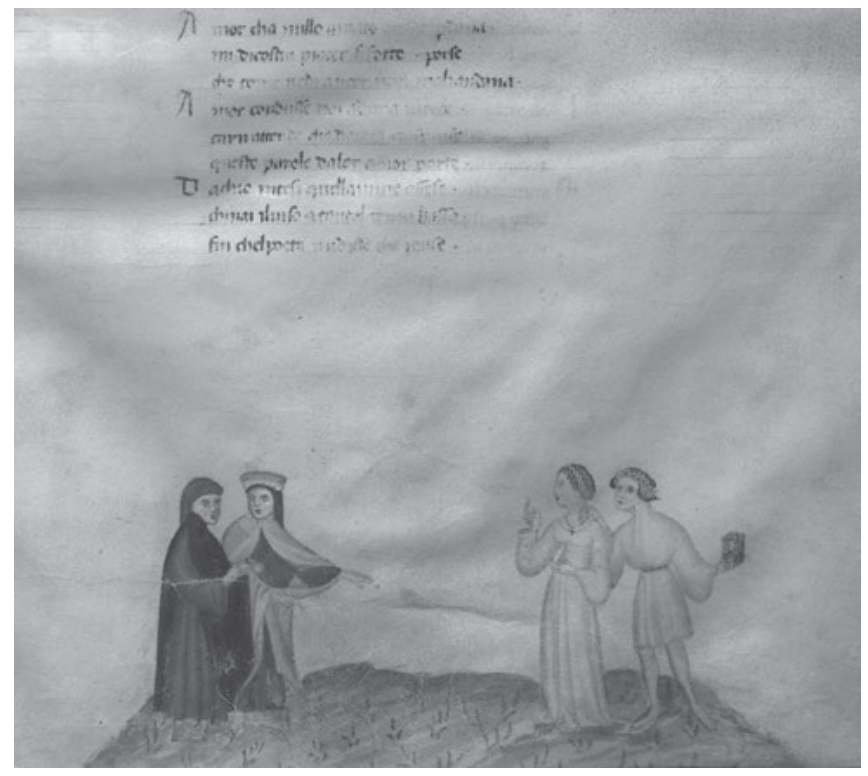

Abb. 3: „Vergil, Dante, Paolo und Francesca“, Miniatur aus der Göttlichen Komödie des Alfons von Aragon, Mitte des XV. Jahrhunderts.

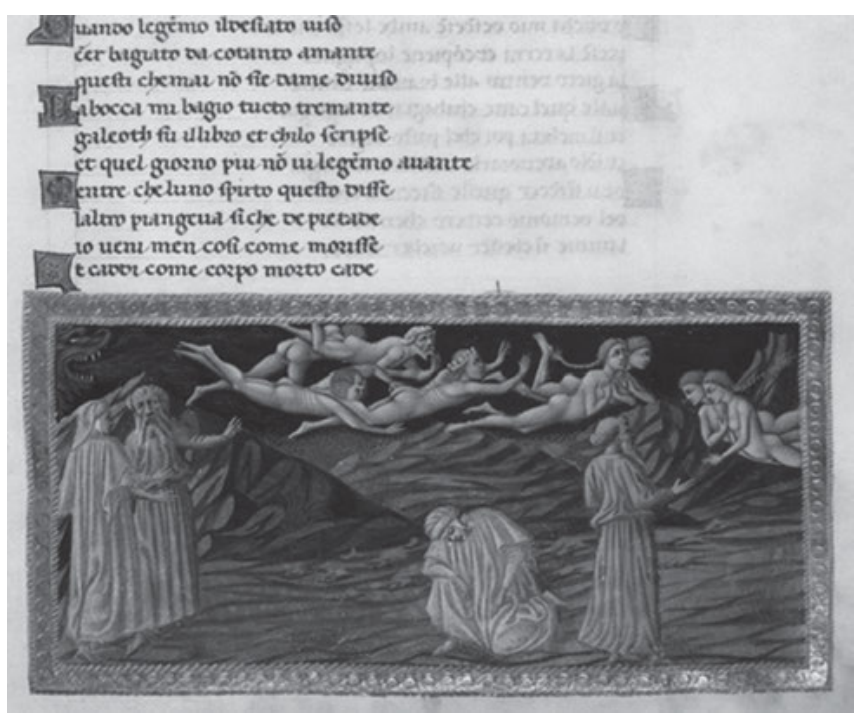

Abb. 4: „Dante und Vergil begegnen Paolo und Francesca“, Miniatur aus Dantes Göttlicher Komödie, Ende des XIV. Jahrhunderts. 
Doch kommen wir wieder zurück zur Lesbarkeit der Welt. Sie wird uns schon bei unserem ersten, eingehender analysierten Beispiel beschäftigen. Wir fragen mit Hans Blumenberg also nicht nur nach dem Verhältnis zwischen Buch und Wirklichkeit, Lesen und Realität, sondern auch nach dem genauen Ort, den das Lesen im Leben einnimmt und wie sein Verhältnis zum Lieben bestimmt werden kann. Tritt das Weltbuch an die Stelle des Buches der Welt und verdrängt es dieses? Oder ist das Lesen doch nur Zeitvertreib, eine Art Totschlagen der Zeit? Für beide Haltungen - und für viele mehr - lassen sich zahlreiche Beispiele aus der Literatur finden, natürlich. In all diesen Fällen übrigens - auch im letztgenannten - stoßen wir auf signifikante Beziehungen zwischen Lesen und Leben. Denn auch das Lesen als Zeitvertreib erfüllt ja eine Funktion gegenüber dem Leben im Leben.

Man könnte aber auch auf eine auf den ersten Blick ganz radikale, absolute Idee verfallen, der ein großer Charme nicht abzusprechen ist: auf jene Idee nämlich, dass man letztlich nur das erkennen und leben kann, was man zuvor bereits gelesen hat. Dies ließe sich mit der Einsicht in der aktuellen Hirnforschung verbinden, dass wir nur scheinbar - und unserem Bewusstsein vorgespiegelt - Entscheidungen treffen. Im Grunde bildet die Literatur für den Fall, dass wir dieses Verständnis des Zusammenhangs von Leben, Lieben und Lesen entwickeln wollten, ein ungeheures kollektives Gehirn, das uns prägt. Wir können dieses Gehirn literaturwissenschaftlich als Intertextualität bezeichnen. Intertextualität ist nicht mehr und nicht weniger als das schlagende Herz der Literaturen der Welt.

Doch all dies wäre gerade in diesem Extremfall nichts weniger und nichts mehr als ein Lebenswissen, ein Wissen, das letztlich auch das Leben von sich selbst hat. Dann aber ist es entscheidend, dieses Gehirn unserer Gesellschaften und Kulturen $\mathrm{zu}$ erforschen. Und Intertextualitätsforschung - also die Erforschung der Bezüge zwischen Texten unterschiedlichster Autorinnen und Autoren - ist eben dies: eine Erforschung jener Graphosphäre - also der Atmosphäre geschriebener Wörter, Sätze und kultureller Praktiken, die wir beständig lesen -, die uns auf geradezu natürliche Weise umgibt, in der wir mit unseren ersten Kinder- und Jugendbüchern aufgewachsen sind und die uns von morgens bis abends begleitet. Es ist die Welt all jener Wörter und Begriffe, all jener Begründungen, vor allem Geschichten, die uns von Kindesbeinen an umgeben haben, noch immer umgeben und künftig auch umgeben werden. Diese Graphosphäre ist kulturell vorgegeben, aber keineswegs ein Fatum, ein Schicksal: Wir können uns in unserem Leben nach und nach unterschiedliche Kulturen und damit unterschiedliche Welten eröffnen und zu eigen machen.

Sicherlich zielen Lehrveranstaltungen stets darauf ab, dass das, was man sich in der Theorie erarbeitet, auch in die Praxis umgesetzt werden kann. Darin 
macht die aktuelle Vorlesung keine Ausnahme. Doch soll dieser Aspekt des Lernens und Erlernens - auch des Erlernens der Liebe selbst - ebenso wenig im Vordergrund stehen wie Versuche, die Liebe als solche $\mathrm{zu}$ definieren und damit zu fixieren. Unsere Vorlesung wird sich also nicht wie jene Serie von Geschenkartikeln, von Tellern, Tassen, Gläsern, Laken oder Bettdecken verhalten, auf denen vor Jahrzehnten - aber bis heute nicht ganz aus der Mode gekommen - Definitionen von Liebe zu lesen waren. Liebe ist: Und es folgten drei Pünktchen und die jeweilige Antwort.

Insoweit muss ich Sie also gleich enttäuschen: Sie werden nicht mit einer griffigen Definition von Liebe diesen Hörsaal verlassen. Ich hoffe aber, dass ich Ihnen andere Geschenke mitgeben kann - denn schließlich waren es ja Sie, waren es die Studierenden der Romanistik, die sich eine Vorlesung zum Thema Liebe gewünscht hatten. Und ich bin dankbar dafür, diesem Wunsch nachgehen $\mathrm{zu}$ können. Wir lernen Liebe so, wie wir rechnen, schreiben und lesen lernen.

Wenden wir uns nun kurz einem italienischen Autor zu, der immer wieder großen Erfolg damit hatte, Vorstellungen der Antike in unserer Gegenwart und mehr noch in unserem Alltagsleben ausfindig zu machen und darüber ausführlich zu reflektieren. Dabei gibt es auch ein Buch, das sich explizit mit der Liebe beschäftigt. Beim Folgenden handelt es sich um Überlegungen, die aus meiner Lektüre von Luciano De Crescenzos 1991 unter dem Titel Miti dell'Amore erschienenen Buch stammen, das in deutscher Übersetzung unter dem Titel Von der Macht der Liebe vorliegt.

Wenn Luciano De Crescenzo, laut Klappentext der deutschen Ausgabe „der lachende Philosoph aus Neapel“, 8 die Liebesgeschichten der Antike noch einmal nacherzählt, so erlaube ich mir, einfach De Crescenzo in einigen wenigen Punkten meinerseits nachzuerzählen und zu deuten. Dies tue ich, um daraus eine Art Sammelsurium zu gewinnen von Themen und Varianten, auf die wir im Verlauf unserer Vorlesung immer wieder stoßen werden. Haben wir also keine Scheu vor einem italienischen Bestsellerautor, den man im gestrengen deutschen Sinne wohl eher nicht als Philosophen bezeichnen würde!

Allem voran betont De Crescenzo, dass schon die Griechen und nach ihnen die Römer sehr wohl wussten, dass die Liebe eine große Macht ist, mit der fürwahr nicht zu spaßen sei, könne sie doch beglückend, aber auch höchst zerstörerisch sein. De Crescenzo konstatiert in seinem Buch zunächst einmal, dass die Kenntnis der mythologischen Persönlichkeiten und Figuren in unserer Gegenwart

8 De Crescenzo, Luciano: Von der Macht der Liebe. Geschichten aus der Antike. Aus dem Italienischen übersetzt von Bruno Gänzler. München: Goldmann 1998. 
abgenommen, die aber des Denver-Clans zugenommen habe. Doch auch wenn Adonis und Aphrodite abgetreten seien, so werde doch bei ausführlicherem Nachdenken deutlich, dass die griechische Mythologie deswegen aus unserem Denken keineswegs verschwunden sei. Denn schließlich seien die Erzählkerne selbst, sozusagen das mythische Grundlagenmaterial, gleich geblieben und hätten sich nur in andere, zeitgenössische Formen gekleidet.

Solchermaßen gerüstet, dürfen wir zur Kenntnis nehmen, dass Eros (alias Cupido) in der Antike als boshaft, heimtückisch und unbarmherzig gedacht wurde. Also keineswegs jener lieblichen Figur eines gelockten Kindchens entsprach, das liebevoll seine Pfeile verschießt. Stellen Sie sich die Figur von Eros bitte ganz anders - machtvoller, herrschender, wohl auch herrischer - vor. Er verschießt lustvoll seine Pfeile, ja, aber ein Entrinnen gab es nicht. Dabei hatte Eros zwei Sorten von Pfeilen in seinem Köcher: Goldpfeile und Bleipfeile. Die einen erregten die Liebe, die zweiten blockierten sie. Eros ist damit eine allmächtige, ja geradezu eine weltenschöpfende Figur der Antike und nicht das kleine unschuldige Jüngelchen, als das man ihn immer wieder verniedlichte. Wir stellen also fest: Eros ist eine Macht - und keine geringe.

All dies wirft natürlich die Frage auf, ob die Liebe eine Kunst ist oder etwas Natürliches, etwas Instinkthaftes. Immerhin, so dürfen wir hier einschieben, ist unser Körper ja nicht nur aus Natur gemacht, wie unsere Schulmediziner meinen, sondern auch aus Kultur: In unserem Körper selbst überschneiden sich Natur und Kultur so intensiv, dass wir in dieser Verbindung durchaus die Grundlage für eine generellere Sichtweise nicht nur der menschlichen, sondern auch der irdischen, der planetarischen Natur erblicken können. Natur ist eben von Kultur nicht zu trennen: beide sind unauflöslich miteinander verbunden.

Stets betrachten wir die Natur von der menschlichen Kultur aus. Dies ist für uns gleichsam natürlich: Wir haben keine andere Wahl. Aber natürlich ist Natur nicht natürlich. Gewiss: Die abendländische Kultur unterschied die Natur von der Kultur nicht nur als voneinander getrennte Einheiten, sondern setzte beide mehr noch in ein Gegensatzverhältnis. Doch vergessen wir hierbei nicht, dass die altchinesische oder die altjapanische Kultur beispielsweise keine getrennten Begriffe für Natur und Kultur besaß und sehr spät auf Lehnwörter zurückgriff, um das abendländische Schema des Natur-Kultur-Gegensatzes nachzubilden. Beide Kulturen unterschieden die Natur nicht von der Kultur. Wir müssen in diesem Jahrhundert wohl erst mühsam erlernen, dass wir Natur und Kultur nicht als Gegensatz, sondern notwendig zusammendenken müssen.

Wenn wir uns auf dieser Ebene bewegen, so sollten wir sogleich eine Unterscheidung mithinzuziehen, die der lange Zeit eher vergessene Philosoph Helmuth Plessner eingeführt hat. Denn er differenzierte zwischen dem Körper, den wir haben, und dem Leib, der wir sind. Diese Aufteilung ist höchst folgenreich und 
wir sollten uns kurz mit dem Spiel zwischen Körper und Leib beschäftigen. ${ }^{9}$ Das Körper-Haben (having a body) zielt auf die Objektivierung des Körpers, meint also einen Körper, den wir besitzen, über den wir als Objekt, als Gegenstand, verfügen und den wir reparieren, schmücken, bemalen, tätowieren oder kosmetisch verschönern können. Wir piercen uns oder rupfen uns Haare und Härchen aus, behandeln unseren Körper folglich als ein Objekt, das wir wie einen Gegenstand bearbeiten. Das Leib-Sein (being a body) wiederum meint den eigentlichen Leib, der wir sind und in dem wir etwa Schmerz oder auch Lust empfinden, über den wir nicht als Objekt verfügen, sondern den wir als Subjekte durchleben. Wie gesagt: Die Folgen dieser schlichten Unterscheidung zwischen Körper-Haben und Leib-Sein sind sehr weitreichend, denken Sie etwa an die Verbindungen zwischen Leib-Sein und Körper-Haben beim Liebesakt, in welchem sich beide Bereiche immer wieder anders durchdringen und in dem wir ständig zwischen Körper-Haben und Leib-Sein oszillieren.

Aber zurück zu De Crescenzo und seiner Deutung der griechischen Mythologie sowie deren Übersetzung in eine italienische, ja neapolitanische Alltagswelt, die noch immer von den Vorstellungen der Antike tief durchdrungen sei. Hier also erscheint Eros als eine Macht, der wir nicht entgehen können, die uns beherrscht und lenkt, ohne dass wir sie wirklich beeinflussen oder gar umstoßen könnten. Wir sind also zum Lieben verdammt. Denken Sie dabei etwa an Romeo und Julia von William Shakespeare, die zwei unterschiedlichen, miteinander tief verfeindeten Adelsgeschlechtern entstammen und doch nicht anders können, als sich zu lieben, als sich im anderen zu verlieren und dabei alles zu verlieren. Das ist in etwa die Vorstellung, die es hier aufzurufen gilt, wollen wir begreifen, welche unbeirrbare, ja unangreifbare Rolle Eros zukommt.

Damit wäre die Frage, ob Liebe eine Kunst ist oder etwas Natürliches, Instinktives, schon beantwortet. Es ist natürlich beides, denn es macht keinen Sinn, an unserem Körper Natur und Kultur zu unterscheiden: wir sind stets beides - und wir sind auch in der Liebe beides, Natur und Kultur.

Für De Crescenzo ist Ovids Ars Amatoria natürlich das beste Handbuch zur Liebeskunst des perfekten Latin Lover. So wird aus hoher Literatur ein Stückchen Alltagskultur. Und so kann man es auch sehen. Es handelt sich folglich auch um eine Kunst, die erlernt werden will, wie es schon zu Beginn dieses Werkes heißt. Auf diese Weise lernen wir mit Ovid viel über das antike Rom und seine herausragenden Orte, an denen Römer und Römerinnen miteinander anbandeln konnten

9 Vgl. hierzu ausführlich Krüger, Hans-Peter: Das Spiel zwischen Leibsein und Körperhaben. Helmuth Plessners Philosophische Anthropologie. In: Deutsche Zeitschrift für Philosophie (Berlin) XLVIII, 2 (2000), S. 1-29. 
und sich anschließend in ihrer Liebeskunst vervollkommneten. Dass dies freilich im Rahmen einer patriarchalisch organisierten Gesellschaft vonstattenging, ist keine Frage und zeigt sich auch im Verlauf von Ovids Werk mit aller Deutlichkeit. Daher rät er gerade den Männern, bei ihren Schönen nicht vor den wildesten Versprechungen zurückzuschrecken: Wir erfahren hier die Tricks und Kniffe, die nicht nur in der Antike angesagt waren, sondern offenkundig auch eine prospektive Dimension bewahren. Oder täusche ich mich?

Die Männer versprechen, die Frauen lügen bei Ovid. Doch man erlernt auch die Kunst, wie man den jeweiligen Liebespartner längerfristig an sich binden kann. Dabei dürfen in der patriarchalischen Gesellschaft seiner Zeit die Männer ihre Frauen betrügen, nicht aber umgekehrt. Freilich sei es nicht klug, die Geliebte mit einem Anderen in flagranti zu ertappen; auch dürfe man den schönen Mädchen ihre Fehler nicht vorhalten. Aber Sie sehen schon: Hier kommen wir in Gefilde, die von unseren heutigen Gewohnheiten wahrlich meilenweit entfernt sind, nicht wahr? Ovid führt uns deutlich vor, wie die Liebeskunst zugleich in den Kodex der Benimmregeln seiner Zeit integriert ist. Der römische Dichter erkennt also die Macht von Eros an, gibt der menschlichen Liebe aber doch jenen Spielraum, in dem sich Tipps, Tricks und Kniffe für ein erfülltes Liebesleben ansiedeln.

Bevor wir uns nun aber Hals über Kopf in unseren Gegenstand, den Themenbereich „Liebe / Lesen“, stürzen, möchte ich Ihnen noch etwas begreiflich zu machen versuchen, vor welchem literatur- und kulturtheoretischem Hintergrund ich Ihnen dieses Thema näherbringen möchte. Dabei haben Sie vielleicht schon bemerkt, dass ich hier einen Begriff eingeführt habe, der Sie vielleicht etwas überrascht hat: den des Lebenswissens. Dieser Begriff bildet den eigentlichen Hintergrund für diese, vielleicht aber auch für künftige Vorlesungen, die sich anderen Phänomenen des Lebens wie Krankheit oder Gesundheit, Eifersucht oder Tod widmen könnten und - in the long run - wohl auch einmal widmen werden. Was aber ist unter dem Begriff Lebenswissen zu verstehen? Zur Beantwortung dieser Frage greife ich auf Überlegungen zurück, die ich in meiner Trilogie ÜberLebensWissen erläutert habe. ${ }^{10}$ Dass das Liebeswissen von Ovid sich sehr wohl als ein Lebenswissen verstehen lässt, leuchtet - so glaube ich - unmittelbar ein. Aber was genau ist unter dem Begriff „Lebenswissen“ zu verstehen?

Nun, dieser Begriff beruht auf einer komplexen Relation zwischen den beiden semantischen Polen dieses Kompositums. Die Interpretierbarkeit dieser Relation als genitivus obiectivus, partitivus, possessivus, qualitatis und nicht zuletzt als genitivus subiectivus verdeutlicht, dass ebenso ein Wissen über das

10 Vgl. Ette, Ottmar: ÜberLebensWissen I-III. Drei Bände im Schuber. Berlin: Kulturverlag Kadmos 2004-2010. 
Leben wie ein Wissen des Lebens von sich selbst, ebenso ein Wissen als wesentlicher Bestandteil des Lebens (und Überlebens) wie eine fundamentale Eigenschaft von Leben überhaupt, ebenso ein Wissen zum Leben wie ein Wissen im Leben mitgedacht und in diesen komplexen Begriff einmontiert sind. Man kann aus dieser Perspektive verschiedene Dimensionen des Lebenswissens voneinander unterscheiden; doch wollen wir uns in dieser Vorlesung nicht mit diesen Differenzierungen aufhalten, sondern uns auf das beziehen, was unmittelbar hinsichtlich der Thematik Liebe und Lesen zielführend ist.

Lebenswissen erscheint gerade mit Blick auf diesen Themenkomplex als ein je spezifischer Modus von Lebensführung und Lebenspraxis, kann als modellhafte Vorstellung wie als beschreibende Aneignung von Leben verstanden werden, wobei die Selbstreferentialität und Selbstreflexivität aller Prozesse des Lebenswissens von herausgehobener Bedeutung ist. Ovids Ars Amatoria lässt sich in diesem Sinne als eine Konkretisierung dieses allgemein verfügbaren, aber jeweils individuell und subjektiv gebrochenen Lebenswissens begreifen, wobei sich der römische Dichter in seinen Recherchen zu seinem Lehrgedicht bemühte, weit über den subjektiv-individuellen Bereich seines eigenen Liebeswissens hinauszugreifen.

Vor diesem Hintergrund und in unterschiedlichen kulturellen Kontexten ist Lebenswissen sehr unterschiedlich strukturiert, insoweit sich Dynamik, Mobilität, Diskontinuität und Fragmentiertheit von Beständen des Lebenswissens immer auch am Flexibilitätsgrad und der Stärke multi-, inter- und transkultureller Prozesse orientieren. Lebenswissen ist hier an spezifische Lebenserfahrungen, nie aber an eine einzige Logik zurückgebunden; vielmehr ist in diesem Begriff gerade die (Überlebens-)Fähigkeit enthalten, gemäß verschiedener Logiken zugleich denken und handeln zu können, kurzum: polylogisch zu reflektieren und $\mathrm{zu}$ agieren.

Unser Lebenswissen wird jedoch nicht nur durch konkrete Erfahrungen in unmittelbaren realen Lebenskontexten, sondern auch durch die Produktion und Konsumption symbolischer Güter, durch die unterschiedlichsten Rezeptionsund Aneignungsformen von Kunst und Literatur gewonnen. Ohne hier auf die Frage nach der Legitimität und Gültigkeit von Überzeugungen, Haltungen und Handlungen, Prinzipien und Praktiken eingehen zu können, bleibt doch festzuhalten, dass es in den nachfolgenden Sitzungen unserer Vorlesung immer wieder um die spezifische Wirkkraft von Literatur gehen wird, die als ein Wissen über Leben und ein Wissen im Leben zugleich ein ÜberLebenswissen bereithält, dessen sich die Figuren unserer ausgewählten Texte bedienen können. Dabei ist die unhintergehbare Vorläufigkeit allen Wissens vom und im Leben, aber auch die ganze Vieldeutigkeit und Fragmenthaftigkeit im Eigen-Leben, im Eigen-Sinn der Literatur geborgen. 
Aus diesen Überlegungen folgt nicht zwangsläufig, dass man in der Literatur eine Art „höheres Lebenswissen“ erblicken müsste, das dem Lebenswissen in unserer Realität überlegen wäre. Wohl aber kommt den Literaturen der Welt das Vermögen zu, normative Formen von Lebenspraxis und Lebensvollzug nicht nur in Szene zu setzen, sondern auch performativ im ernsthaften Spiel zur Disposition und möglichen Aneignung zu stellen. Das Literarische enthält stets ein Wissen um die Grenzen der Gültigkeit von Wissensbeständen einer gegebenen Gesellschaft oder Kultur und thematisiert dies auch, wird folglich selbstreflexiv. Untersuchen wir Leseprozesse, so untersuchen wir Aneignungsprozesse oder -versuche dieses Wissens, die - wie wir noch sehen werden - historisch und kulturell hochgradig variabel sind. Dabei können Lesen und Lieben gleichermaßen im Fokus des Lebenswissens stehen. Denn das Lesen ist wie das Lieben, soviel können wir jetzt schon sagen, keine anthropologische Konstante, sondern ein hochgradig variabler Prozess, der sich je nach historischem oder kulturellem Kontext sehr stark verändert.

Vor diesem Hintergrund und mit allen notwendigen, von diesen Kontexten abhängigen Differenzierungen darf Literatur in ihren unterschiedlichsten Schreibformen als ein sich wandelndes und zugleich interaktives Speichermedium von Lebenswissen verstanden werden. Anders als in der Philosophie geht es freilich im Bereich des Literarischen nicht um die Konstruktion in sich kohärenter logischer Sinnsysteme, sondern um die künstlerische Fähigkeit, Kohärenzen durch Dekohärenzen - worunter man in der Quantentheorie Überlagerungszustände und Superpositionen versteht - zu bereichern. Polysemie war noch immer der Schlüssel zu den Literaturen der Welt.

Es ist die Aufgabe der Philologie, sich mit diesen spezifischen und höchst unterschiedlichen Traditionen, Genres, Dimensionen und Ausprägungsformen von Lebenswissen auseinanderzusetzen. Die Philologien würden sich dadurch in die Lage versetzen, ihrerseits Formen und Modi von Lebenswissen zu produzieren, die gesellschaftlich, politisch und kulturell relevant und bedeutungsvoll werden könnten. Sie würden damit Alternativen zu Lebensoptionen oder auch politischen Entscheidungen offerieren, um einer zunehmend phantasielos gewordenen Welt der Politik Alternativen bieten zu können.

Zweifellos benötigen wir eine grundlegende Einsicht in die Tatsache, dass sich Lebenswissen auf allen Ebenen der literarischen Kommunikation ansiedelt. Dabei steht gerade nicht ein vermeintlich „direkter“ Dialog zwischen literarischem Text und textexterner Leserschaft im Vordergrund. Von vorrangigem Interesse könnten gerade jene Bestände an Lebenswissen sein, die sich auf die textinterne Kommunikationssituation beziehen und beispielsweise die verschiedenen Figuren eines Romans mit sehr unterschiedlichen Formen von Lebenswissen (etwa mit Blick auf Liebesgeschichten oder Leseprozesse) 
ausstatten. Dabei sollten wir keinesfalls die herausragende Fähigkeit der Literaturen der Welt vergessen, in den unterschiedlichsten kulturellen und sozialen Kontexten die dispersen Fragmente des Lebenswissens in ein Überlebenswissen zu verwandeln, welches nicht nur von textinterner Bedeutung ist. Auch dieses Wissen kann von der Leserin und vom Leser beansprucht werden: Lesen kann das Leben nicht nur bereichern, es kann auch zum eigenen Überleben beitragen. Selbstverständlich können bestimmte Wissenssegmente als Gnoseme von Überlebenswissen angeeignet werden. Ganz so, wie wir auch den anderen Fall kennen, bei dem die Lektüre letztlich zum Tod führt - denken wir nur an die Selbstmordwelle nach der Lektüre von Goethes Werther. Und nicht umsonst ist bei diesem literarischen Beispiel Liebe und Lesen ganz eng miteinander verbunden. Aber dazu später mehr.

Literatur - in einem weiten Sinne verstanden - richtet ihren Lebensbegriff weder vorrangig an einer Scheidung von Organischem und Anorganischem, weder allein an leiblichen oder körperlichen, seelischen oder geistigen Dimensionen von Leben aus. Sie verfügt über viele Codes, über unterschiedlichste Denk- und Schreibtraditionen, die in ihrer Vielgestaltigkeit, aber auch in ihrer Aussagekraft mit den Ergebnissen aktueller biowissenschaftlicher Forschungen in Beziehung gesetzt werden können und sollten. Denn wie naturwissenschaftliche Forschung beruht auch die Praxis der Literatur auf Recherche, auf der Erforschung jeweils definierter Gegenstandsbereiche. Im Unterschied zu den Naturwissenschaften aber ist die Literatur nicht an die Herrschaft einer einzigen Logik gebunden, sondern kann sehr wohl polylogisch vorgehen, sich folglich in verschiedenen Logiken zugleich bewegen. Selbst in den traditionellsten Geisteswissenschaften beginnt die Überzeugung Raum zu greifen, dass der menschliche Körper nicht mehr nur aus motivgeschichtlicher Sicht erforscht und ansonsten als „Natur“ den medizinisch-naturwissenschaftlichen Fakultäten überlassen werden kann. Die Entwicklung neuer Formen inter- und transdisziplinärer Zusammenarbeit ist zum Erwerb neuen Wissens über Leben, Lesen und Lieben dringend geboten. Eben dies ist die Chance der Literatur und mehr noch ihrer Philologien.

Es kann folglich nicht darum gehen, Lebenswissen, wie es sich in textueller Form ästhetisch ausdrückt, ausschließlich disziplinär zu erfassen und damit zu disziplinieren. Der Begriff des Wissens - und damit auch jener des Lebenswissens - übersteigt den Bereich der Wissenschaft und schließt insbesondere künstlerische, narrative, poetische und körperleibliche Wissens-, Ausdrucksund Speicherformen mit ein, die ihrerseits wissenschaftlicher Analyse zugänglich sind oder doch gemacht werden können. Dabei sind in den Literaturen der Welt unterschiedlichste Formen narrativen Wissens eine wesentliche Grundlage des entfalteten Lebenswissens. Ich möchte daher an dieser Stelle auf eine Geschichte, auf eine künstliche Mythe, die ich im Eröffnungsband meiner Trilogie 
ÜberLebensWissen behandelt habe, ${ }^{11}$ zurückgreifen, um noch einen weiteren Aspekt von Lebenswissen in seiner Verbindung mit der Liebe und mit dem Lesen herauszuarbeiten.

Unsere Geschichte spielt im Mittelmeer und selbstverständlich in der Antike. Dem Ende seines Lebens nahegekommen, erhält Epicharmus von Dionysius den Befehl, ein vielberufenes Bild zu deuten, um das sich das Volk von Syracus seit langer Zeit versammelt. Allen Erklärungsversuchen zum Trotz war das Rätsel jenes Bildes, dem man nachträglich den Titel Der rhodische Genius gegeben hatte, noch immer ungelöst geblieben. In diesem Werk eines unbekannten Künstlers hält, von einer Gruppe unbekleideter Jünglinge und Mädchen umgeben, ein jugendlicher, fast noch kindlicher Genius mit himmlischem Blick eine lodernde Fackel empor und schaut gebieterisch auf die zu seinen Füßen versammelte sehnsuchtsund kummervoll zugleich wirkende Jugend herab. Erst als ein aus Rhodos in den Hafen von Syracus eingelaufenes Schiff dem Tyrannen ein Gemälde überbringt, das denselben rätselhaften Genius inmitten seiner Gruppe zeigt, nun aber mit gesenktem Haupt, erloschener Fackel und umgeben von Mädchen und Jünglingen, die sich im Zustand wilder Entfesselung umarmen, wird das Bedürfnis nach Deutung unerträglich: Epicharmus, der Philosoph, soll das Rätsel lösen - und sie sehen angesichts der ineinander liebevoll vereinigten Körper von Mädchen und Jünglingen unschwer, wie viel diese Mythe mit dem Thema Liebe zu tun hat. Aber auch mit dem Themenkomplex des Lesens: Denn die Aufgabe des Philosophen - und des Philologen - besteht genau darin, die beiden Bildtexte zu lesen und damit ihre Rätselstruktur zu lösen.

Der Philosoph und Philologe ist dabei erfolgreich. Er vermag es, die Gründe für die enthemmte Orgie auf der symbolischen Ebene zu entschlüsseln. Auch wenn der Wahrheitsfreund fernab vom Hofe lebt, der selbst den Geistreichsten stets von ihrem Geist und ihrer Freiheit raubt, weiß er dabei doch um seine Pflichten gegenüber der Macht. So schart er, wie Philosophen es zu tun pflegen, seine Schüler um sich und enthüllt ihnen nach intensiver Betrachtung beider Gemälde das Geheimnis. Des Rätsels Lösung ist dabei eine durchaus komplexe Lesart beider Gemälde: Der rhodische Genius symbolisiert die Lebenskraft, die anders als in der anorganischen Natur gebieterisch im Organismus all jene Elemente und Stoffe vereinigt, die sich sonst meiden, um zugleich jene anderen voneinander fernzuhalten, die ohne die Lebenskraft miteinander verschmelzen, ineinander stürzen und vergehen müssten. Epicharmus ist sich seiner Sache sicher und führt aus:

11 Vgl. die Eröffnung von Ette, Ottmar: ÜberLebenswissen. Die Aufgabe der Philologie. Berlin: Kulturverlag Kadmos 2004. 
Tretet näher um mich her, meine Schüler, und erkennet im rhodischen Genius, in dem Ausdruck seiner jugendlichen Stärke, im Schmetterling auf seiner Schulter, im Herrscherblick seines Auges das Symbol der Lebenskraft, wie sie jeden Keim der organischen Schöpfung beseelt. Die irdischen Elemente, zu seinen Füßen, streben gleichsam ihrer eigenen Begierde zu folgen und sich mit einander zu mischen. Befehlend droht ihnen der Genius mit aufgehobener, hochlodernder Fackel, und zwingt sie, ihrer alten Rechte uneingedenk, seinem Gesetze zu folgen. ${ }^{12}$

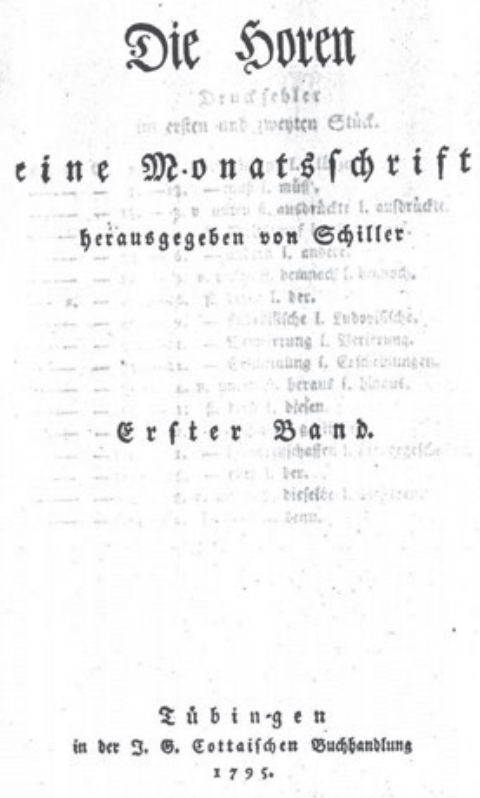

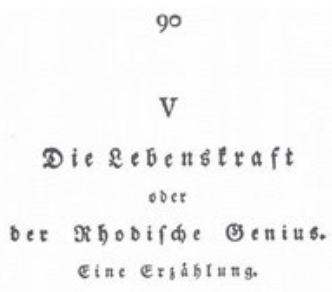

Die Evratufer batten ifgren \$oitile wie bie eatbenet. Borfelfungen von Gòttern unb seroen, griedbifdoe unb ttalifide sunftwerte betleibeten bie bunten satten bes Đortifub̧. Unabláśg fạ man oas Bolt babin ftròmen, ben jungen אrieger, um fid) an ben इbaten ber 9tbnberrn, ben Sünfter, um fid) an bem Vinfel grefier פreifter zu weiben. Unter ben jabllofen Semábloen, meldee ber emfige Fleif ber Gyratufer aus bem Drutterlanbe gefam. melt; war nur einç, bab feit eincm bollen 3abrbunberte

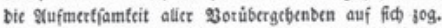
2Benu es Dem Dlpmpifłen Jupiter, Dem Etábtegrúnber Eetrops, bem şclbenmutb bes sparmebius unb 2triftogi. ton an Berwunbercen feblte, fo fand bod um jenes Bild bas Bolt in bidten Motten gebrángt. \$ober biefe 2ot. liebe füt baffelbe? \$at es ein geretteted $23 e r t$ bes Apelo

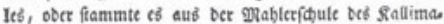

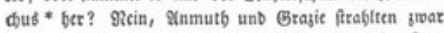
aus bem SBilde bervor, aber an $3 e r f d$ melgung ber fat.

- Cacizotechnos. Plia. XXXIV. 19. a. 3 s.

Abb. 5a und b: Titelblatt der Zeitschrift Die Horen und des Beitrags von Alexander von Humboldt, 1795.

So erweist sich die Orgie, das Übereinanderfallen der noch jugendlichen Körper von jungen Männern und jungen Frauen, in Wahrheit als eine symbolhafte Darstellung des Erlöschens der Lebenskraft, also des Todes schlechthin. Im zweiten Bild fehlt die vis vitalis, das alles belebende Prinzip. Der Philosoph von Syracus durfte sich seiner Sache sicher sein, hatte ihm doch Alexander von Humboldt nach eigenem Bekenntnis Lehrsätze zur Lebenskraft aus der Physiologie der

12 Humboldt, Alexander von: Die Lebenskraft oder der rhodische Genius. Eine Erzählung. In (ders.): Ansichten der Natur, mit wissenschaftlichen Erläuterungen. Nördlingen: Greno 1986, S. $429 \mathrm{f}$. 
Pflanzen in den Mund gelegt, die er selbst 1793 in den „Aphorismen“ seiner Flora Fribergensis in lateinischer Sprache hatte abdrucken lassen. Epicharmus scheint nichts anderes als das Sprachrohr der Wissenschaft zu sein: einer Wissenschaft freilich, die sich in das Gewand griechischer Mythologie kleidet.

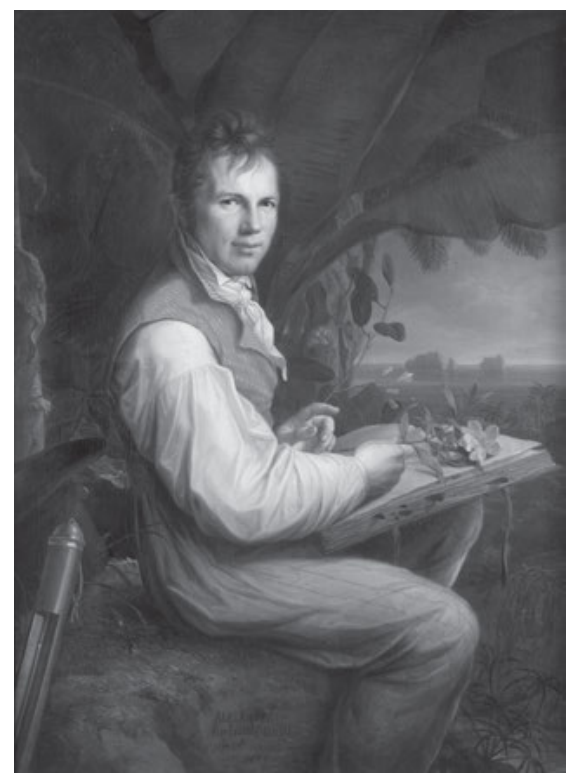

Abb. 6: Alexander von Humboldt (Berlin, 1769 - ebenda, 1859).

Als Alexander von Humboldt in Friedrich Schillers Horen 1795 die kleine Erzählung zum ersten Mal erscheinen ließ, bewegte sie sich folglich auf naturwissenschaftlich gesichertem Terrain. Zwei Jahre später aber, am Ende seiner Versuche über die gereizte Muskel- und Nervenfaser - die er als „ein großes Werk über das Leben“ bezeichnete ${ }^{13}$ - war sich der junge Wissenschaftler seiner Sache längst nicht mehr so sicher, hielt er doch nun „das Vorhandensein jener eigenen Lebenskräfte keineswegs für erwiesen“. ${ }^{14}$ Wir befinden uns an einem wichtigen Übergang der Wissenschaftsgeschichte, der die Verbannung der vis vitalis aus dem Bereich der Wissenschaft, nicht aber aus der metaphorischen, übertragenen Rede oder auch der Alltagssprache bezeugt.

Es erstaunt daher zumindest auf den ersten Blick, dass Alexander von Humboldt seine Erzählung, die sein älterer Bruder Wilhelm von Humboldt kurzerhand

13 Humboldt, Alexander von: Briefe aus Amerika 1799-1804. Herausgegeben von Ulrike Moheit. Berlin: Akademie Verlag 1993, S. 63.

14 Humboldt, Alexander von: Ansichten der Natur, S. 432. 
den „halbdichterische[n] Einkleidungen ernsthafter Wahrheiten“ zurechnete, ${ }^{15}$ Jahrzehnte später in die 1826 erschienene zweite Ausgabe seiner Ansichten der Natur (sogar als Schlusstext) aufnahm und auch in der dritten, nochmals erweiterten Auflage von 1849 seine Jugendschrift nicht missen wollte. War dies die Nostalgie eines alten Mannes, der auf die achtzig Jahre seines Lebens und mehr als sechs Jahrzehnte erfolgreicher wissenschaftlicher Arbeit zurückblicken konnte?

Nun, zunächst liegen die Gründe für den erneuten Abdruck tatsächlich wohl in einer Form der Selbstliebe, einer Wertschätzung dessen, was er selbst vor so langer Zeit geschrieben hatte und in dem er sich selbst noch immer trefflich spiegeln konnte. In der Tat enthält die Erzählung durch die Zusätze von 1849, in denen Alexander von Humboldt auf seine frühesten Schriften verweisen, aber auch mehrfach aus seiner wissenschaftlichen Summa, dem Kosmos, zitieren konnte, (s)eine Wissenschaftlerbiographie in nuce. Und nicht minder musste manche Passage ein eigentümliches Licht auf den als Kammerherrn am preußischen Hofe lebenden Schriftsteller und Gelehrten werfen, ließ der Erzähler von Epicharmus sein zeitgenössisches Publikum doch wissen:

Er besuchte selten den Hof der Dionyse: nicht, als hätten nicht ausgezeichnete Männer aus allen griechischen Pflanzstädten sich um ihn versammelt, sondern weil solche Fürstennähe auch den geistreichsten Männern von ihrem Geist und ihrer Freiheit raubt. Er beschäftigte sich unablässig mit der Natur der Dinge und ihren Kräften, mit der Entstehung von Pflanzen und Thieren, mit den harmonischen Gesetzen, nach denen Weltkörper im großen, und Schneeflocken und Hagelkörner im kleinen sich kugelförmig ballen. ${ }^{16}$

Nicht allein vor dem Hintergrund des preußischen Königshofes in Berlin und Potsdam, sondern auch angesichts der Tatsache, dass Alexander von Humboldt mit den beiden preußischen Königen, denen er als Kammerherr diente, ein freundschaftliches Verhältnis verband, besitzt der Text etwas Schillerndes - gerade auch mit Blick auf den Zeitpunkt seiner Wiederveröffentlichung in den Ansichten der Natur. Die Beispiele für die Präsenz des eigenen Lebens, der autobiographischen Dimension seiner Erzählung, ließen sich trotz der Kürze des Textes leicht mehren.

Und doch tritt in dieser Schrift neben der naturwissenschaftlichen und der autobiographischen Dimension des Lebens noch eine weitere hinzu, die das Leben des Textes selbst betrifft und die Humboldt zum späten und wiederholten Abdruck seines frühen literarischen Versuchs bewegt haben dürfte. Der naturwissenschaftlichen Legitimation, der allegorischen Behauptung einer

15 Alexander zitiert in seinem auf März 1849 datierten Vorwort zur zweiten und dritten Ausgabe der Ansichten der Natur aus dem Brief seines Bruders (Ansichten der Natur, S. 10).

16 Humboldt, Alexander von: Ansichten der Natur, S. 428. 
wissenschaftlich angenommenen Existenz von Lebenskräften, war längst die Grundlage entzogen; an autobiographischen Passagen war im Humboldt'schen Gesamtwerk kein Mangel. Die Gründe für die Aufnahme des kurzen Textes in seine Ansichten der Natur, in denen sich Literatur und Wissenschaft miteinander verknüpfen und jene „Verbindung eines litterarischen und eines rein scientifischen Zweckes“ eingehen, die Humboldt so sehr erstrebte, mussten daher andere sein. Fassen wir dies etwas genauer ins Auge.

Diese Gründe dürften jenseits des autobiographischen Charmes zum einen sicherlich im Thematischen liegen. Denn die Beschäftigung mit dem Leben in seinen vielfältigsten wissenschaftlichen und gesellschaftlichen, kulturellen und künstlerischen Formen ist im Kosmos der Humboldt'schen Schriften und Aktivitäten zweifellos von überragender Bedeutung. Alles scheint sich bei Humboldt um den „Prozess des Lebens“ zu drehen: Er war beständig, wie es Ilse Jahn ausdrückte, „dem Leben auf der Spur“. ${ }^{17}$ Allein schon durch ihre bis heute die Leserschaft überraschende Präsenz macht „Die Lebenskraft oder der rhodische Genius“ auf diese Tatsache aufmerksam. Denn der Begriff des Lebens war für alle Schriften Humboldts von zentraler Relevanz.

Zum anderen aber führt der literarische Text selbst vor, wie er sich der vom Philosophen vorgetragenen wissenschaftlichen Begründung ein ums andere Mal entzieht und sein Eigen-Leben entwickelt. Nicht umsonst ist der Titel dieser Erzählung zweigliedrig: Eine allein wissenschaftliche Titelgebung - etwa „Die Lebenskraft“ - hätte nicht ausgereicht, um die verdoppelte Bewegung von wissenschaftlicher und literarischer Logik wiederzugeben. Humboldts Text ist in seiner Vieldeutigkeit bestechend: Er spielt die volle Kraft des Literarischen nicht gegen, aber mit dem Wissenschaftlichen aus. Die Erzählung mag zwar ein Rätsel lösen, gibt aber viele weitere Rätsel auf: Warum wird das Erlöschen der Lebenskraft gerade mit den „mannigfachen Umarmungen“ der nackten Jünglinge und Mädchen verbunden, die sich im „Zustand wilder Entfesselung“ der „Befriedigung lang genährter Sehnsucht“ überlassen? Oder warum ist die Erkenntnis der Lebenskraft mit dem unmittelbar bevorstehenden Tode des Epicharmus verknüpft? Alexander von Humboldts kleine literarische Skizze lässt viele Fragen offen, mit anderen Worten: Sie weist eine hohe semantische Offenheit auf, die von keiner physiologischen Hypothese begrenzt werden kann. Warum ist die Liebe symbolhaft mit dem Tod verbunden? Die literarische Logik lässt sich auf die (natur-)wissenschaftliche nicht reduzieren. Die Proble-

17 Vgl. Jahn, Ilse: „Dem Leben auf der Spur“. Die biologischen Forschungen Alexander von Humboldts. Leipzig - Jena - Berlin: Urania Verlag 1969; sowie dies.: „Dem Leben auf der Spur“. Die biologischen Forschungen Alexander von Humboldts. In: HiN - Alexander von Humboldt im Netz (Potsdam - Berlin) X, 18 (2009), S. 68-95. 
matik des Lebens ist durch Axiome nicht fest-zustellen. Sie entzieht sich und kommt in den Bereichen des Ästhetischen als eines Verbindungswissens zwischen allen Wissenschaften, zwischen allem Wissen, zum umfassenden und vieldeutigen Ausdruck.

Doch Alexander von Humboldts „Rhodischer Genius“ hält für unsere Vorlesung noch weitere bemerkenswerte Aspekte bereit. Denn es ist aufschlussreich, dass in Humboldts Erzählung die Frage nach der Lebenskraft erst auftaucht, als sich an das erste ein zweites Bild anfügt und damit eine - freilich nicht nur linear deutbare - Bewegung im Sinne einer Bilderfolge in den Blick gerät. Diese Bewegung führt zwischen lodernder und gelöschter Fackel narrative Strukturen und nicht auf eine einzige Finalität oder Kausalität reduzierbare Polysemien ein, deren Vieldeutigkeit das Leben als Offenheit textuellen Eigen-Lebens vorführt. Erst die bewegten Bilder bewegen den Philosophen zu seiner grundlegenden Deutung, welche Leben, Lesen und Liebe miteinander in Verbindung bringt und zugleich auf symbolischer Ebene eine lebenswissenschaftliche Tonart anschlägt. So wird deutlich, dass „Die Lebenskraft oder der rhodische Genius“ ein Wissen vom Leben bereithält, das sich gerade nicht auf eine naturwissenschaftliche Theoriebildung vom Leben beschränken und begrenzen lässt. Die ästhetische Dimension des Schreibens meint nichts Ornamentales, sondern ist gerade in der Unabschließbarkeit der von ihr in Gang gesetzten oder noch in Gang zu setzenden Bedeutungsprozesse selbst Wissen vom Leben, in narrativer Form.

Es ist dieses narrative Wissen, das sich in seiner literarischen Vieldeutigkeit mit dem naturwissenschaftlichen Wissen verbindet und eine lebenswissenschaftliche Dimension in diesen frühen und zugleich späten literarischen Text Humboldts einbringt. Und eben darum darf die Literatur, dürfen die Literaturen der Welt als ein sich wandelndes und zugleich interaktives Speichermedium von Lebenswissen verstanden werden. In eben diesem Sinne ist die Literatur eine zutiefst lebenswissenschaftliche Kunst- und Wissensform und bedarf einer lebenswissenschaftlich ausgerichteten Philologie, um sie in ihrer viellogischen und vieldeutigen Strukturierung genussreich zu lesen.

Alexander von Humboldts Erzählung ist ein Grenztext, der die erwähnte Absicht seiner Ansichten der Natur, die „Verbindung eines litterarischen und eines rein scientifischen Zweckes“, in hoher ästhetischer Verdichtung demonstriert und vorführt. In der zweiten Hälfte des 20. Jahrhunderts, vergessen wir dies nicht, sind die Grenzen zwischen den Wissenschaften, aber auch zwischen Wissenschaft und Literatur erneut und in ebenso vielfältiger wie fundamentaler Weise in Bewegung geraten. Feste Grenzziehungen zwischen Natur- und Kulturwissenschaften, Geistes- und Gesellschaftswissenschaften entsprechen zwar noch immer gängigen Ordnungssystemen des Wissenschaftsbetriebs zu Beginn 
des 21. Jahrhunderts, aber längst nicht mehr den Entwicklungen innerhalb einer zunehmend transdisziplinär ausgerichteten Wissenschaftspraxis, für welche auch die Grenzen zwischen Natur und Kultur und damit auch die von Charles Percy Snow beschworenen Two Cultures, brüchig und fragwürdig geworden sind. Bereits zu Beginn der neunziger Jahre des zurückliegenden Jahrhunderts zeichnete sich eine derartige Entwicklung deutlich ab. Es bedarf keiner Sehergabe, um heute prognostizieren zu können, dass sich diese Entwicklung unter dem Eindruck der Entfaltung transdisziplinärer Wissenschaftskonzepte parallel und komplementär zu weiteren Formen von Ausdifferenzierung und Spezialisierung des Wissens im 21. Jahrhundert weiter beschleunigen wird. Unsere Vorlesung versucht, dieser Entwicklung Rechnung zu tragen und die Zusammenhänge zwischen Lieben, Lesen und Leben aus einer stark lebenswissenschaftlich geprägten Perspektive auszuleuchten.

Gewiss ist die Veränderung wissenschaftlicher Paradigmen eine höchst komplexe und in der Regel eher langsam verlaufende Angelegenheit. Gleichzeitig ist diese Transformation in hohem Maße an die Entfaltung wissenschaftlicher Kreativität gekoppelt. Die Erschließung neuer Wissensräume zwischen oder quer zu vorhandenen Einteilungen vorherrschender Wissenschaftssystematik bildet eine der fundamentalen Voraussetzungen für Kreativität und Produktivität, aber auch für Präsenz und Performanz der Wissenschaften in ihrem jeweiligen gesellschaftlichen Umfeld. Doch gibt es nicht selten den Fall, dass neue Wissensräume nicht erschlossen und genutzt, sondern nominell okkupiert werden, ohne dass die beteiligten Wissenschaften in der Lage wären, ein ihrer neu geschaffenen Begrifflichkeit entsprechendes Instrumentarium $\mathrm{zu}$ entwickeln, das den von ihnen selbst ausgespannten Horizont füllen könnte. Nicht selten sind wissenschaftsstrategische Entscheidungen mit derlei Entwicklungen verbunden.

Ein gutes Beispiel hierfür bietet der Begriff der Lebenswissenschaften. Seit das Jahr 2001 von der damaligen Bundesministerin für Bildung und Forschung im Verbund mit wissenschaftlichen Institutionen zum „Jahr der Lebenswissenschaften“ ausgerufen wurde, erweckten die Diskussionen um das menschliche Genom, um die Stammzellenforschung oder um die Möglichkeiten, tierisches oder menschliches Leben zu klonen, Erbgut oder Saatgut gentechnisch zu manipulieren, in der Öffentlichkeit zunehmend den Eindruck, die hier angesprochenen hochspezialisierten Wissenschaften deckten das gesamte Spektrum menschlichen Lebens ab. Dieser Eindruck bei einer breiten Öffentlichkeit war durchaus erwünscht. Feuilletons, Fernsehserien, politische Debatten oder Talk-Shows waren zumindest vor dem 11. September 2001 von der Suche nach dem Schlüssel zum menschlichen Leben beherrscht, wobei man sich Leben mit zunehmender Ausschließlichkeit als einen komplexen, aber entschlüsselbaren Code vorzustel- 
len begann. Derlei Vorstellungen waren öffentlichkeitswirksam inszenierbar. Und sie wurden unendliche Male in Szene gesetzt.

Die sich hieraus ergebenden Konsequenzen waren in ihrer Tendenz eindeutig. Dank faszinierender Verstehensmodelle und beeindruckender Forschungsergebnisse, die mehr und mehr auf Bereiche des Alltagslebens und der Zukunftssicherung durchschlugen, wurden die Biowissenschaften in den Massenmedien, aber auch in der Forschungsförderung zu dem, was über den ursprünglichen Gebrauch des englischsprachigen Begriffs der life sciences deutlich hinausgeht: Sie wurden zu den Wissenschaften vom Leben überhaupt proklamiert. Das Rätsel des Lebens, das Alexander von Humboldt noch als literarisch-philosophisch-naturwissenschaftliche Allegorese gestalten konnte, schien nun entzifferbar: als rechenbare und letztlich berechenbare Kette: als Code direkt und eindeutig lesbar. Doch das Leben ist mehr als ein lesbarer, eindeutig verwertbarer Code.

Dem Universalitätsanspruch und der Hegemonie eines bestimmten Fächerspektrums innerhalb des seit Beginn des 19. Jahrhunderts verschärft ausgetragenen Wettstreits der Wissenschaften sollte man jedoch entgegenhalten, was Hans-Georg Gadamer ausgehend von einer Philosophie des Hörens und Zuhörens zum Verhältnis zwischen Natur- und Geisteswissenschaften ins Feld führte:

Nun pflegt man den Geisteswissenschaften ja gern gerade die Frage zu stellen, in welchem Sinne sie Wissenschaft sein wollen, wenn es kein Kriterium für das Verständnis von Texten oder Worten gibt. Für die Naturwissenschaften und die Verkehrsformen der Technik ist gewiß richtig, daß Eindeutigkeit der Verständigungsmittel garantiert ist. Aber unbestreitbar macht selbst der Apparat einer auf Wissenschaft und Technik gegründeten Zivilisation lange nicht das Ganze des Miteinanderlebens aus. ${ }^{18}$

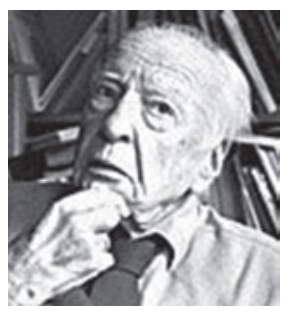

Abb. 7: Hans-Georg Gadamer (Marburg, 1900 - Heidelberg, 2002), in seinem Heidelberger Arbeitszimmer im Jahr 1999.

Vorsicht ist also geboten: Hüten wir uns vor dem Alleinvertretungsanspruch bestimmter Wissenschaften, die doch nur ein ganz bestimmtes und von ihnen definiertes Feld des Wissbaren zu erkunden vermögen. Das Abstecken bestimm-

18 Gadamer, Hans-Georg: Über das Hören. In: Vogel, Thomas (Hg.): Über das Hören. Einem Phänomen auf der Spur. Tübingen: Attempto Verlag 1996, S. $202 \mathrm{f}$. 
ter begrifflicher Claims sollte uns dabei nicht davon abschrecken, die Polylogik der Geistes- und Kulturwissenschaften gerade auf dem Gebiet des Wissens vom Leben im Leben geltend zu machen. Der Begriff der Lebenswissenschaften ist nicht nur so vieldeutig und schillernd, so umfassend und marktgängig, als wäre er von Werbestrategen eigens für die Durchsetzung biowissenschaftlichnaturwissenschaftlicher Interessen in Sozial- wie Forschungsgemeinschaften konzipiert; er ist überdies ein Verdrängungsbegriff, der nicht nur den Begriff vom Leben im Vergleich zur abendländischen Antike ungeheuer reduziert, sondern durch seine besitzergreifende Tendenz andere Wissenschaften gleichsam vom Zugang zum Leben fernhält - und dies gerade wegen seiner Nutzbarmachung einer der Literatur und den Geisteswissenschaften entlehnten Metaphorik. Nicht nur der genetische Code des Lebens, sondern auch jener der Inszenierung der Biowissenschaften ist in einem kritischen und wissenschaftspolitisch aufgeklärten Sinne lesbar. Wir sollten folglich den neuen Mythen der Natur- und Biowissenschaften nicht aufsitzen und auch deren Terminologie stets hinterfragen.

Die unterschiedlichen Geistes- und Kulturwissenschaften haben disziplinär jeweils verschieden auf diese Herausforderungen reagiert. So hat die Philosophie längst - dies ist aus der Geschichte dieser Disziplin selbstverständlich - im Bereich der Eugenik die Frage nach einem Leben ohne „das Bewegende von moralischen Gefühlen der Verpflichtung und der Schuld, des Vorwurfs und der Verzeihung, ohne das Befreiende moralischer Achtung, ohne das Beglückende solidarischer Unterstützung und ohne das Bedrückende moralischen Versagens, ohne die ,Freundlichkeit‘ eines zivilisierten Umgangs mit Konflikt und Widerspruch" gestellt. ${ }^{19}$ Doch ist die Tragweite des biowissenschaftlichen Griffes in die semantische Trickkiste in jenen Wissenschaften, die sich doch vordringlich mit semantischen Verfahren beschäftigen, noch kaum reflektiert worden. Dies aber scheint mir überfällig.

Wenn es denn Lebenswissenschaften in einem dem Begriff adäquateren Sinne geben soll, müssen sie das breite Spektrum des griechischen bíos (und nicht nur zoé) und damit auch die unterschiedlichen Logiken, die sich in der Beschäftigung mit verschiedenartigsten Bereichen des Lebens ausgebildet haben, integrieren. Es wird folglich in dieser Vorlesung darum gehen, gerade auch die kulturellen Dimensionen des Lebensbegriffs - und damit meine ich auch implizit die multi-, inter- und vor allem transkulturellen Dimensionen - einzubeziehen und für eine fruchtbare Analyse bereitzuhalten. Leben ist per se viellogisch und nicht gemäß

19 Habermas, Jürgen: Die Zukunft der menschlichen Natur. Auf dem Weg zu einer liberalen Eugenik? Vierte, erweiterte Auflage. Frankfurt am Main: Suhrkamp 2002, S. 124. 
einer disziplinären Logik - und sei es die eines Fächerensembles - strukturiert. Das „nackte“, das „bloße“ Leben, das den Menschen mit allen anderen Lebewesen verbindet, und das politisch, sozial und kulturell geprägte Leben - und damit „Natur“ und „Kultur“ in ihrem Lebensbezug - sind gemäß unterschiedlichster Logiken relational aufeinander zu beziehen. Denn wir haben ja bereits gesehen, dass wir Natur und Kultur nicht länger simplistisch voneinander trennen dürfen.

Auf der Ebene des Lebenswissens überschneiden sich Kultur- und Naturwissenschaften, bilden Heterotopien des Wissens, die wir nicht einfach dem einen oder dem anderen Bereich allein zuordnen und damit monosemieren können. Als Horizontbegriff stellt Lebenswissen disziplinäre Grenzziehungen in Frage und verlangt nach transdisziplinären Herangehensweisen, welche Wissensbestände der Literatur- wie der Gesellschaftswissenschaften, der Kultur- wie der Naturwissenschaften mit dem sich verändernden Gedächtnis der Literaturen der Welt verbinden. Die kulturellen Praktiken des Lesens wie auch die großen Emotionen der Liebe sind dem Lebenswissen wie den breit verstandenen Lebenswissenschaften zugänglich und erschließen in ihrer Verknüpfung neue, kreativ zu eröffnende Bereiche des Wissens. Die Einsicht, „daß Rationalität plural ist“,20 bietet eine gute Grundlage, um unterschiedliche Logiken, verschiedene Kulturen, Künste und Wissenschaften vielstimmig zu Wort und zu Gehör kommen zu lassen. Dass unser Schwerpunkt als Philologinnen und Philologen dabei auf den Literaturen der Welt liegt, versteht sich von selbst.

Literatur erschließt Lebenswissen narrativ nicht zuletzt als Erlebenswissen. Dies betrifft gerade den Themenkomplex sehr deutlich, in welchem wir uns zwischen Liebe und Lesen bewegen. Dieses Erleben ist als Erlebnis wie als Nacherleben ohne jeden Zweifel wissenschaftlicher Analyse zugänglich, sei sie produktions- oder rezeptionsästhetischer Ausrichtung. Die Narration des großen Gefühls der Liebe und deren rezeptionsbedingte Aneignung durch eine Leserschaft stellt Fragen nach einer Erzeugung und Generierung wie nach den Tradierungs- und Aneignungsformen von Liebe in unserer aktuellen Gesellschaft wie auch in vorgängigen, historisch gewordenen Gesellschaftsformationen. Vielleicht liegt hierin das Vermächtnis der postum veröffentlichten Vorlesung Roland Barthes' am Collège de France, die der Frage des Zusammenlebens in Differenz und damit einer zutiefst lebenswissenschaftlichen Problematik gewidmet ist. Denn nicht umsonst hatte auch Hans-Georg Gadamer im oben angeführten Zitat auf die Notwendigkeit eines „Miteinanderlebens“, einer Konvivenz der

20 Daston, Lorraine: Wunder, Beweise und Tatsachen. Zur Geschichte der Rationalität. Aus dem Englischen von Gerhard Herrgott, Christa Krüger und Susanne Scharnowski. Frankfurt am Main: Fischer Taschenbuch 2001, S. 11. 
Wissenschaften, hingewiesen. Dies gilt auch und gerade für ein transdisziplinär konzipiertes Zusammenspiel unterschiedlicher Wissenschaften, welches für unsere Vorlesung von entscheidender Bedeutung ist, will sie ihre Themenstellung vielperspektivisch angehen.

Zugleich sollen auch Bereiche eines Lebenswissens erfasst werden, die sich noch nicht stabilisiert haben und im Grunde noch über keine (wissenschaftstheoretischen) Diskurse und folglich keine diskursive Existenz verfügen, auch wenn sie von den Künsten, Literaturen und Medien längst präsentiert und repräsentiert werden. Hierzu zählt auch und gerade der diskursive Bereich der Liebe, der - das wird Sie vielleicht überraschen - als einer der absoluten Einsamkeit bezeichnet worden ist. Dem werden wir nachgehen, keine Sorge!

Leben und Lust, Körper und Wissen, Spielformen literarischen Schreibens und Schreibformen literaturwissenschaftlichen Spiels sollen bei der Findung und Erfindung neuer Wissensräume in die Konstruktion wissenschaftlicher Objekte eingehen und zugleich deren wissenschaftliche Subjekte bereichern und verändern. Die Aufgabe der Philologie als treue Freundin des (literarischen) Wortes und als Wissenschaft mag in der Zukunft nicht unwesentlich davon abhängen, wie sie ihr Verhältnis zum Leben und zur Liebe - und damit natürlich auch zum Tod - bestimmt.

Nun, diese Überlegungen sollten Sie zunächst einmal mit einer gewissen wissenschaftsgeschichtlichen, aber auch methodologischen und forschungsstrategischen Logik konfrontieren, die dem Vorhaben zu Grunde liegt, das wir heute gemeinsam miteinander in Angriff nehmen. Zugleich aber sollte Ihnen auch klargeworden sein, dass man den Begriff Liebe - ebenso wenig wie den Begriff Leben - auf biowissenschaftliche Dimensionen reduzieren darf; denn dann wird aus dem Leben wie aus der Liebe das Funktionieren eines bestimmten Apparats, den ich mechanistisch oder gentechnologisch, aber doch im Grunde aus der Logik der abendländischen Wissenschaften heraus letztlich als eine Maschine oder ein von wem auch immer aufgezeichnetes Programm und das Abspulen dieser programmierten Abläufe verstehen kann. Liebe aber ist auch und keineswegs zuletzt eine hochdifferenzierte diskursive Semantik und eine kulturell gesteuerte Ästhetik, die ihre pragmatische Seite nicht verleugnen kann.

Damit rücken - wie das von uns kurz untersuchte Humboldt'sche Beispiel zeigt - intermediale Übersetzungsformen von Wissen, aber auch performative Aspekte gerade mit Blick auf die Lebenspraxis und ein anzustrebendes „gutes Leben“ in den Vordergrund. Ein Wissen von der Liebe kann dabei ebenso in Schrifttexten (etwa im Roman oder einer philosophischen Lebens- oder Liebeslehre, in Biographien oder Autobiographien, in philologischen Reflexionen oder moralistischen Maximen) wie in Bildtexten, in der Inszenierung und Performanz des Körper-Leibs wie in der Befragung und (künstlerischen oder wissenschaftlichen) 
Repräsentation von Körperwissen hergestellt, dargestellt und gedeutet werden. Wir werden uns mit all diesen unterschiedlichen Gattungen und Textsorten in unserer Vorlesung befassen. Gerade den Spielformen eines narrativen Wissens werden wir uns dabei besonders zuwenden, sind in ihrem Erzählen von Geschichten doch zugleich auch Handlungsmöglichkeiten und Handlungsanweisungen versteckt, die sehr wohl auf eine jeweilige Leserschaft Einfluss nehmen können. Auch hier ist wiederum die Art der Aufnahme dieses Wissens das Lesen.

Besteht aber nicht die Gefahr, Literatur und Kunst - mithin „sekundäre modellbildende Systeme“,21 von denen Jurij M. Lotman geradezu liebevoll sprach - mit dem „Leben“ und damit das Lesen von der Liebe mit dem Erleben der Liebe gleichzusetzen, einer kruden Widerspiegelungstheorie zu huldigen oder in einen schematischen Realismus prästrukturalistischer wie präpoststrukturalistischer Provenienz zurückzufallen?

Eine klare Trennung zwischen textinternen und textexternen Kommunikationsebenen in den nachfolgenden Untersuchungen dürften derartige Befürchtungen zusammen mit einer jeweiligen soziohistorischen und kulturellen Kontextualisierung der analysierten Texte ausräumen. Es gilt, eine Vielzahl unterschiedlicher Perspektivierungen unseres Forschungsgegenstandes - also des Wissens von der Liebe - zu erproben und auszutesten. Die wissenschaftliche Beschäftigung mit einem Wissen von der Liebe kann die Vermittlung von Erlebenswissen ebenso miteinschließen wie Erörterungen eines Wissens vom Zusammenleben verschiedener Kulturen, ebenso Fragen von Leiblichkeit oder Körperlichkeit erörtern wie wissenschaftliche oder pseudowissenschaftliche Abhandlungen über diese Themenkomplexe untersuchen. Die Lust am Text wird dabei die Bereiche von Liebe und Lesen stets miteinander $\mathrm{zu}$ verbinden trachten.

Doch was haben Leben und Liebe mit dem Lesen, mit dem Akt der Lektüre zu tun? Eine fundamental-komplexe Sichtweise von Leben ließe sich auch auf jenes fundamental-komplexe System der Literatur übertragen, das so spielerisch mit den Problematiken von Reversibilität und Prognostizierbarkeit umzugehen versteht und uns zugleich Anfang und Ende des Lebens - die unserem bewussten Erleben als Menschen entzogen sind - in der verlebendigenden Kraft des Leseakts zugänglich macht. Es geht beim Lesen folglich immer um die Sehnsucht nach einer Ganzheit von Leben und Liebe, um die Herstellung jener Totalität, die uns Menschen im realen Leben grundlegend entzogen ist, können wir uns doch weder an den Vorgang unserer eigenen Zeugung oder unserer eigenen Geburt, also an unsere Anfänge, noch an unser eigenes Ende selbstreflexiv erinnern. Allein die

21 Lotman, Jurij M.: Die Struktur literarischer Texte. Übersetzt von Rolf-Dietrich Keil. München: Fink - UTB ${ }^{2} 1981$, S. 23. 
Literatur erlaubt es uns, über die Totalität eines Lebens mit seinen Anfängen und mit seinen Enden zu verfügen. Und genau dies gilt auch und gerade für die Liebe und deren Geschichten und Vorgeschichten. Noch das Scheitern einer Liebesbeziehung und damit des Zusammenlebens mit dem geschlechterspezifisch, kulturell, religiös oder ethnisch Differenten setzt in der Literatur ein Wissen frei, das sie für ihr Lesepublikum als komplexes, nicht auf simple Handlungsanweisungen reduzierbares Wissen vom Leben und von der Liebe bereithält. Um eben dieses Wissen geht es uns.

Lesen und Schreiben sind fundamentale Kulturtechniken des Menschen, und sie sind dabei auf eine grundlegende Komplementarität hin angelegt. Dies gilt in vielfacher Hinsicht: ebenso mit Blick darauf, dass das Geschriebene durch Lesen angeeignet wird, wie auch, dass das Gelesene seinerseits wieder eingeht in ein Schreiben, das nur - wie wir noch sehen werden - auf den ersten Blick Teil einer rein schriftlichen, nicht-mündlichen Kommunikation ist. Doch Schreiben und Lesen, Lesen und Schreiben verkörpern auch höchst unterschiedliche Gewichtungen von Wissen, wie dies Michel de Certeau in einer Passage festhielt, die von Roger Chartier und Guglielmo Cavallo in ihrem lesenswerten Buch Die Welt des Lesens an den Anfang gestellt wurde. Sie haben damit an den Anfang ihres Schreibens ein Gelesenes gesetzt, so wie ich meinerseits auf dieses von ihnen Gelesene als ein Geschriebenes zurückgreife, das ich wiederum meinerseits zum Lesen gebe, ohne dabei freilich die beiden Lektüreforscher zu vergessen. Doch hören wir erst dieses Zitat des französischen Philosophen Michel de Certeau:

\footnotetext{
Weit davon entfernt, Schreibende zu sein, Gründer einer Heimstatt auf den Erblanden der Sprache, Bauern der Vergangenheit, Bohrer von Brunnen und Erbauer von Häusern, sind Leser Reisende: Sie ziehen wie Nomaden durch fremdes Gebiet, wildern auf Feldern, die nicht ihre Handschrift tragen, und plündern alle Schätze Ägyptens, um sich daran zu erfreuen. Das Schreiben häuft an, lagert ein, widersteht der Zeit durch die Schaffung einer Heimstatt und vermehrt seine Erzeugnisse durch eine auf Expansion bedachte Vervielfältigung. Das Lesen sichert sich nicht ab gegen den Verschleiß durch die Zeit (man vergisst sich und man vergisst es), es bewahrt das Erworbene nicht oder nur schlecht auf, und jeder Ort, an dem es vorbeikommt, ist eine Wiederholung des verlorenen Paradieses. ${ }^{22}$
}

22 Certeau, Michel de: L’invention du quotidien, Bd. 1, Arts de faire, Paris: Gallimard, S. 251. Soweit nicht anders angegeben, stammen alle Übersetzungen ins Deutsche vom Verfasser. Die originalsprachigen Zitate finden die Leserin und der Leser in den Anmerkungen. 


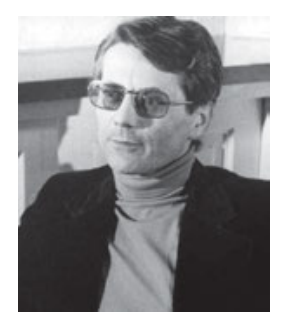

Abb. 8: Michel de Certeau (Chambéry, 1925 - Paris, 1986).

Zweifellos: Literatur steht in einer ganz fundamentalen Beziehung zu einem Paradies, genauer: zu einem verlorenen Paradies. ${ }^{23}$ Doch laut Michel de Certeau ergibt sich hier vor allem eine Art Opposition zwischen dem Schreiben und dem Lesen, die wie eine Art Zweitaktmotor unseres kulturellen Systems im Abendland funktionieren. Das Schreiben ist sesshaft und zielt auf Sammlung und Lagerung, das Lesen hingegen ist nomadisch und zielt auf Umherziehen, Plünderung und ständige Wiederholung eines immer wieder von neuem vergessenen oder verlorenen Paradieses.

Man könnte hier vieles anmerken, auf was wir auch im Folgenden noch kommen werden: etwa die Tatsache, dass auch das Lesen - schon etymologisch gesehen - anhäuft und vor allem sammelt, was freilich nicht notwendigerweise $\mathrm{zu}$ einer sesshaften Lebensweise führen muss. Lesen aber ist - wie man es im Deutschen noch mit dem Begriff der Weinlese vor Augen führen kann - mit dem Sammeln, der Sammlung verbunden, ohne die das Lesen nun einmal nicht auskommt. ${ }^{24}$ Selbstverständlich sind auch wir Literaturwissenschaftlerinnen und Literaturwissenschaftler Sammler, die ihre Schätze in privaten Bibliotheken anhäufen, vor allem aber ihre Sammlungen im Kopf ständig pflegen.

Wichtig an Michel de Certeaus Bemerkung aber scheint mir auch die Verbindung zu sein, die zwischen dem Lesen und dem Reisen hergestellt wird, eine Verbindung, die sich sicherlich auch auf der Ebene der Bewegung auf dem Papier - in unserer Kultur von links oben nach rechts unten, aber auch springend, hüpfend, hin- und herblätternd - nachzeichnen lässt. Lesen ist in einem fundamentalen Sinne mit dem Reisen verbunden - und zwar nicht nur in der Reiseliteratur, für welche die Beziehung zwischen Reisen und Schreiben grundlegend ist.

Doch die Verbindung zwischen dem Lesen und dem Reisen ist tiefgründig, bedeutet das Lesen doch stets den Besuch neuer Orte, die besichtigt werden, die Auseinandersetzung mit neuen Wissensinhalten und Wissensformen, die

23 Vgl. Ette, Ottmar: Konvivenz. Literatur und Leben nach dem Paradies. Berlin: Kulturverlag Kadmos 2012.

24 Vgl. Sánchez, Yvette: Coleccionismo y literatura. Madrid: Ediciones Cátedra 1999. 
zugänglich gemacht werden, die Aneignung von etwas Fremdem, das gleichsam inkorporiert wird. Wenn wir etwas von Liebe lesen, inkorporieren wir folglich etwas, das uns potenziell irgendwann als ein Handlungsmuster dienen kann oder als ein abzulehnendes Gegenmodell erscheint, das wir weit von uns weisen können.

Die Verbindung zwischen der Lesemetaphorik und der Reisemetaphorik lässt sich quer durch die Literaturen der Welt nachweisen. Und nicht zuletzt steht auch am Anfang des abendländischen modernen Romans, in Miguel de Cervantes' Don Quijote, die Reise, die auch immer ein Lesen der Welt auf der Hintergrundfolie des Lebens darstellt. Das Gelebte wird aus dem Gelesenen bezogen, und dieses Gelesene zwingt - wie beim Ritterroman - zur ständigen Bewegung: ebenso im Leben wie beim Lesen. So sammeln wir folglich bei der Lektüre Handlungsmuster, Handlungsformen und Handlungsnormen im Bereich gerade auch der Liebe.

Nun aber ist es an der Zeit, dass wir uns mit einem ersten literarischen Text, einem ersten Roman, beschäftigen. Er soll uns als eine Art Vorspiel dienen, als ein Beispiel, an dem wir vorab genau jene Beziehung untersuchen können, welche im Zentrum unserer Vorlesung steht: die vielfältige Relationalität zwischen Lesen und Lieben.

Machen Sie sich also auf ein erstes „Schmankerl“, einen literarischen Leckerbissen, gefasst. Denn ich möchte diese für die Literaturen der Welt konstitutive Beziehung gerne mit Ihnen am Beispiel eines der großen - nicht nur italienischen - Romane der zweiten Hälfte des 20. Jahrhunderts näher überprüfen. Anhand dieses Exempels werde ich Ihnen exemplarisch vorführen, worum es im Kern in dieser Vorlesung geht - und die Lust am Text ist dabei garantiert. 